\title{
COMMUNICABLE DISEASES REPORT, NSW, FOR OCTOBER AND NOVEMBER 2003
}

\section{TRENDS}

Notifications of communicable diseases through midspring indicated a decline in influenza, invasive pneumococcal disease and meningococcal disease (Figure 2, Tables 1-2). Because the surveillance case definition for pertussis requires, in part, the patient to have a coughing illness for 14 days, there is an inherent delay between onset of disease and notification of the case. Recent trends in case reports of pertussis are therefore likely to be substantially underestimated, and it is possible that a further rise in case reports will occur in coming months.

A large gastroenteritis outbreak caused by Norovirus infection was identified in October, involving over 70 people in the Greater Murray Area Health Service. The most likely cause of this outbreak was contamination of food by a food handler. Norovirus is infectious with low doses of the virus, which can survive on surfaces and in foods for long periods. People who are ill with gastroenteritis should stay home and not prepare food for anyone until 48 hours after their symptoms have completely resolved. A report of this outbreak will be published in a future issue of the NSW Public Health Bulletin.

For updated information, visit www.health.nsw.gov.au and click on the link to Infectious Diseases.

\section{INFLUENZA SURVEILLANCE 2003}

Robin Gilmour, Clayton Chiu, and David Muscatello Enhanced surveillance for influenza in NSW indicates that the 2003 influenza season peaked in August. Most cases were caused by the influenza A virus, predominantly the A/Fujian/411/2002 strain. Little influenza B infection was reported. Preliminary analysis of emergency department data, supported by anecdotal reports from clinicians, suggests that influenza may have affected more people in 2003 than in previous recent years.

In 2003, several sources of data were included in an enhanced surveillance for influenza, including:

\section{Sentinel general practitioners}

Up to 48 general practitioners participated in weekly reporting of influenza-like illness (ILI) activity. ILI activity peaked in mid August (34.7 per 1,000 consultations). In 2002, a similar peak (36.6 per 1000 consultations) appeared in July of that year.

\section{Virological surveillance}

Six sentinel laboratories tested 10,391 respiratory samples for the presence of influenza virus, by either direct immunofluorescence (DIF) or culture. Influenza A was found in 831 samples, and this strain peaked in mid-tolate August (23.2 per 100 samples). In 2002, detection of influenza A peaked at 16.9 per 100 samples. Influenza B was found in 13 samples, and this strain peaked in early September (0.5 per 100 samples).

\section{Serological surveillance}

The same six sentinel laboratories tested 4,052 serum samples for evidence (seroconversion or rise in IgG level or high single titre) of infection with influenza. Serological diagnoses of influenza A peaked in early September, at 13.6 per 100 samples. In 2002, a similar peak (14.1 per 100 samples) appeared in late August. Serological diagnoses of influenza B were rare and no peak was identified. In 2002, the peak occurred in early July (7.8 per 100 samples).

\section{General practitioner direct virological surveillance}

In 2003, fifteen general practitioners (GPs) volunteered to provide specimens from patients who they suspected to have influenza infection for virological testing. Threehundred-and-nine samples were taken by the GPs, of which 51 (16.5 per cent) were positive for influenza A. No samples tested positive for influenza B.

\section{The WHO Influenza Collaborating Centre}

The WHO Influenza Collaborating Centre for Reference and Research on Influenza, located in Melbourne, reports that the majority of influenza $\mathrm{A}$ isolates identified during the peak period were $\mathrm{A}(\mathrm{H} 3)$ viruses of the $\mathrm{A} / \mathrm{Fujian} / 411 /$ 2002 type. This year, some antigenic drift has been detected in the virus strains circulating in Australia and New Zealand. The A/Fujian-like viruses are related to the A/Moscow-like strain included in the 2003 vaccine, and the vaccine has been demonstrated to induce antibodies to the A/Fujian-like strains but generally at a reduced level. In the last few years, dominant strains of influenza A have included A/Nanchang/95, A/Sydney/97, A/Moscow/99, and A/New Caledonia/99 (the last part of the name of each strain represents the year in which it was first identified).

\section{Emergency department surveillance}

Information on visits to NSW Emergency Departments (EDs) collected routinely by hospitals is currently being evaluated as a monitoring tool for influenza surveillance. Figure 1 compares the number of ED visits assigned a provisional diagnosis of influenza in hospitals participating in the NSW Emergency Department Data Collection with other influenza indicators currently used in NSW. Only EDs providing reasonably-complete provisional diagnosis information using the International Classification of Diseases for the period July 1996 to October 2003 were included. The collection captures approximately two-thirds of NSW Emergency Department visits. Peaks in the number of visits to EDs assigned a provisional diagnosis of influenza corresponded to peaks in reports from laboratory virology and GP sentinel surveillance. The highest peaks in the years 1997, 2000, 


\section{FIGURE 1}

COMPARISON OF EMERGENCY DEPARTMENT VISITS FOR INFLUENZA-LIKE ILLNESS WITH LABORATORYBASED DETECTION RATES AND GENERAL PRACTITIONER SENTINEL SURVEILLANCE FOR INFLUENZA, NSW, 1996-2003
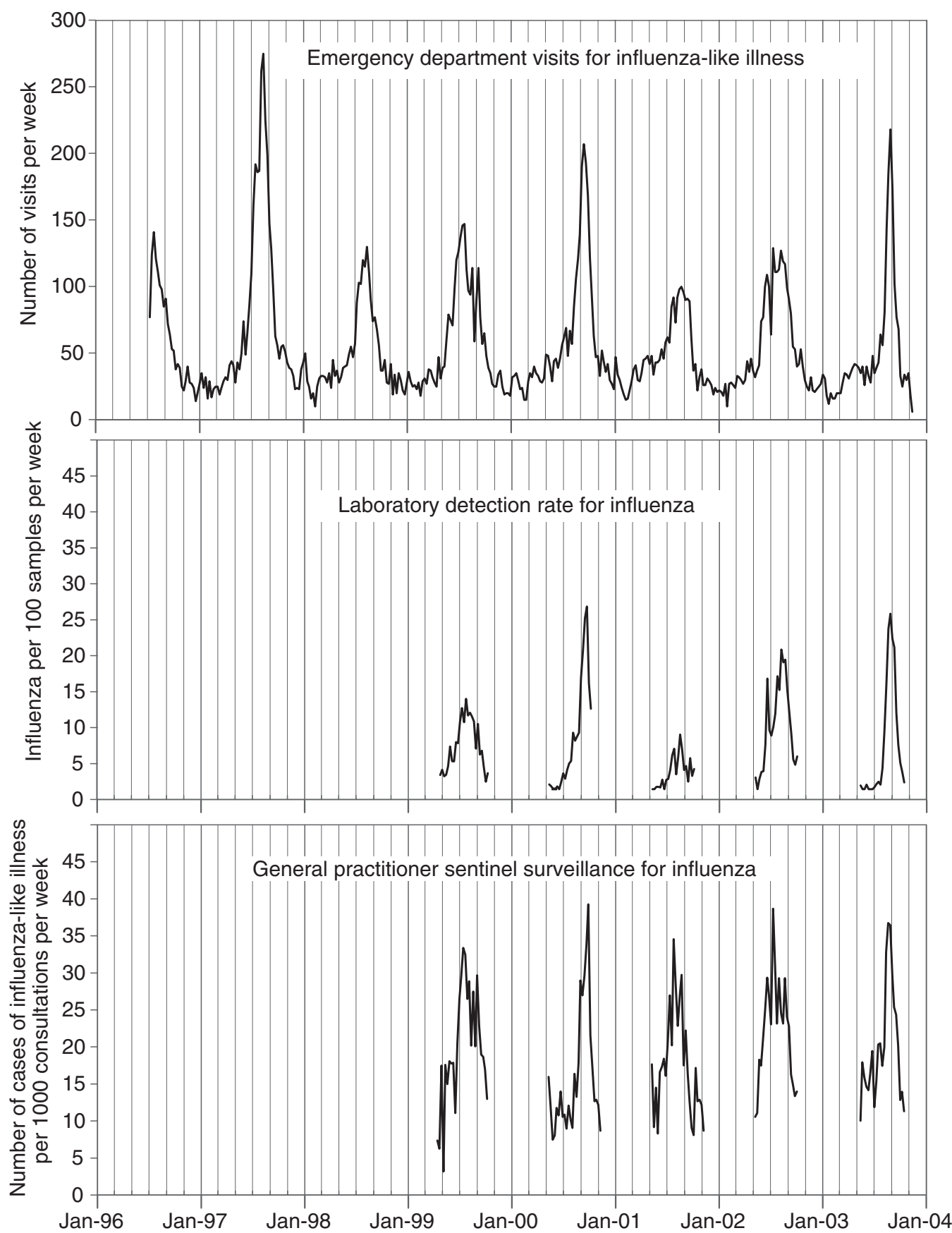

Notes: Influenza-like illness in emergency departments was based on unplanned visits assigned a principal provisional diagnosis of influenza. Laboratory and general practitioner data were only available from May 1999.

Source: NSW Emergency Department Data Collection (HOIST), Centre for Epidemiology and Research, NSW Department of Health; and NSW Influenza Surveillance Program, Communicable Diseases Branch, NSW Department of Health. 
and 2003 coincided with a predominance of newly emergent strains of influenza A virus among laboratory samples that had strain identification performed (Sydney/ 97, Moscow/99 and Fujian/2002 in each of those peak years respectively).

\section{COMMENT}

The data and information collected from these sources indicate that influenza peaks each year in winter, usually between mid-July and mid-September. In 2003, the peak was in August, and influenza activity may have been more widespread than in recent previous years.

There are several limitations to these data. First, none of the surveillance systems mentioned here are very sensitive: all collect data on only a very small proportion of people infected with influenza in NSW, and this proportion may vary over time, rendering comparisons open to bias. Second, none of the systems provide a very representative sample of influenza cases either by the demographics of the affected people, their place of residence, or severity of illness. Laboratory surveillance is based in urban hospitals, and is be more likely to include very sick children (who tend to present to hospital for testing) than the GP systems. Participating GPs are not located randomly across the state. Third, apart from the laboratory-based systems, the diagnosis of influenza-like illness is not specific, and the systems are likely to pick up a range of other respiratory conditions not caused by influenza viruses.

The apparent triennial variation in the magnitude of the influenza peaks found in Emergency Department visits appears to be temporally associated with the predominance of a newly emergent A strain among circulating strains of the influenza virus for the year. These data suggest that ED surveillance could be a useful tool for monitoring not only the occurrence of influenza epidemics in NSW but also their extent. The Centre for Epidemiology and Research has developed methods for the rapid transfer and analysis of these data for surveillance purposes. 


\section{FIGURE 2}

\section{REPORTS OF SELECTED COMMUNICABLE DISEASES, NSW, JANUARY 1996 TO NOVEMBER 2003,}

BY MONTH OF ONSET

These are preliminary data: case counts for recent months may increase because of reporting delays. Laboratory-confirmed cases, except for measles, meningococcal disease and pertussis.

\begin{tabular}{|rc|}
\hline \multicolumn{2}{|c|}{ NSW population } \\
Male & $50 \%$ \\
$<5$ & $7 \%$ \\
$5-24$ & $28 \%$ \\
$25-64$ & $52 \%$ \\
$65+$ & $13 \%$ \\
Rural $^{*}$ & $42 \%$ \\
\hline
\end{tabular}
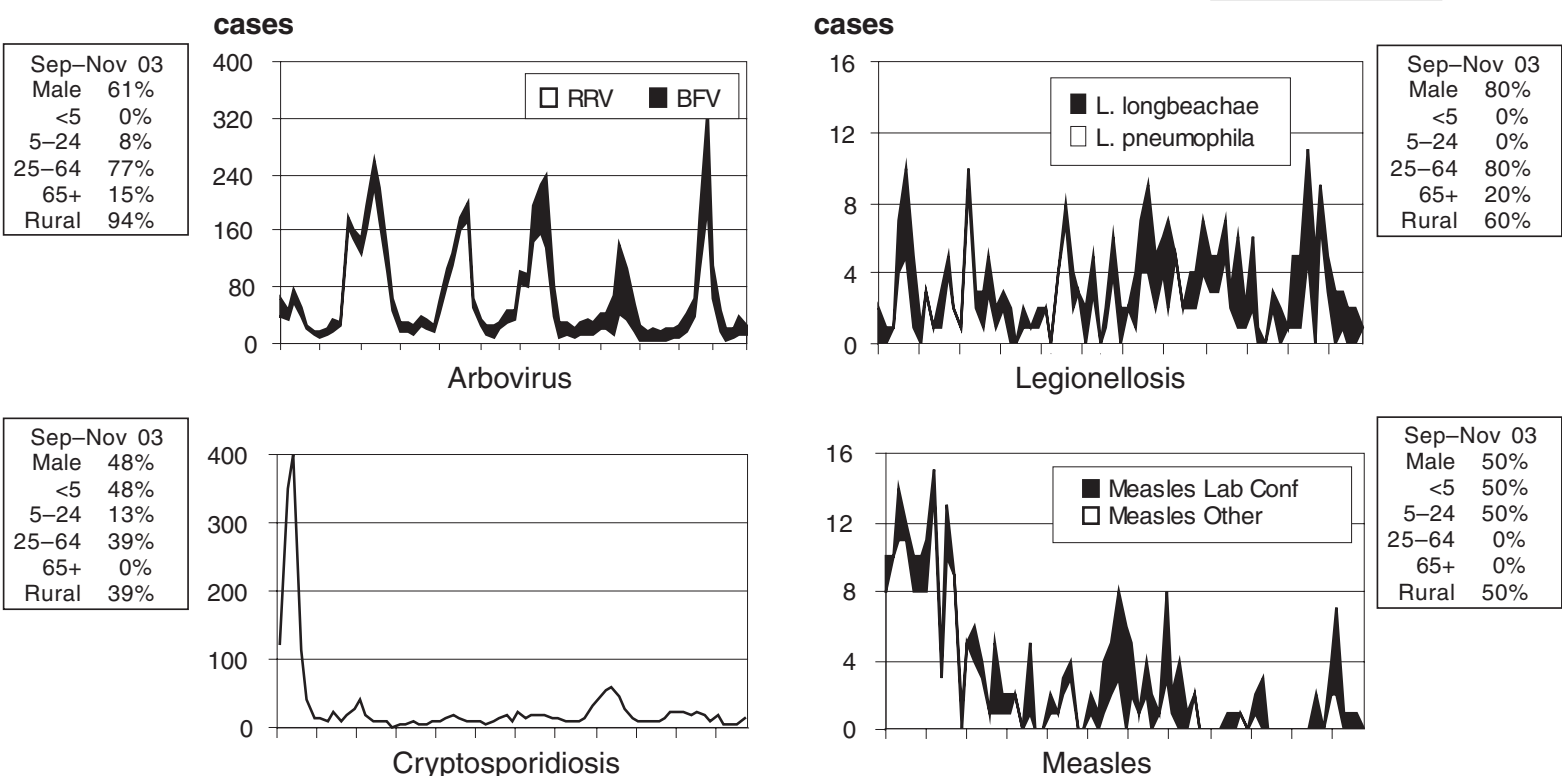

Cryptosporidiosis

Measles
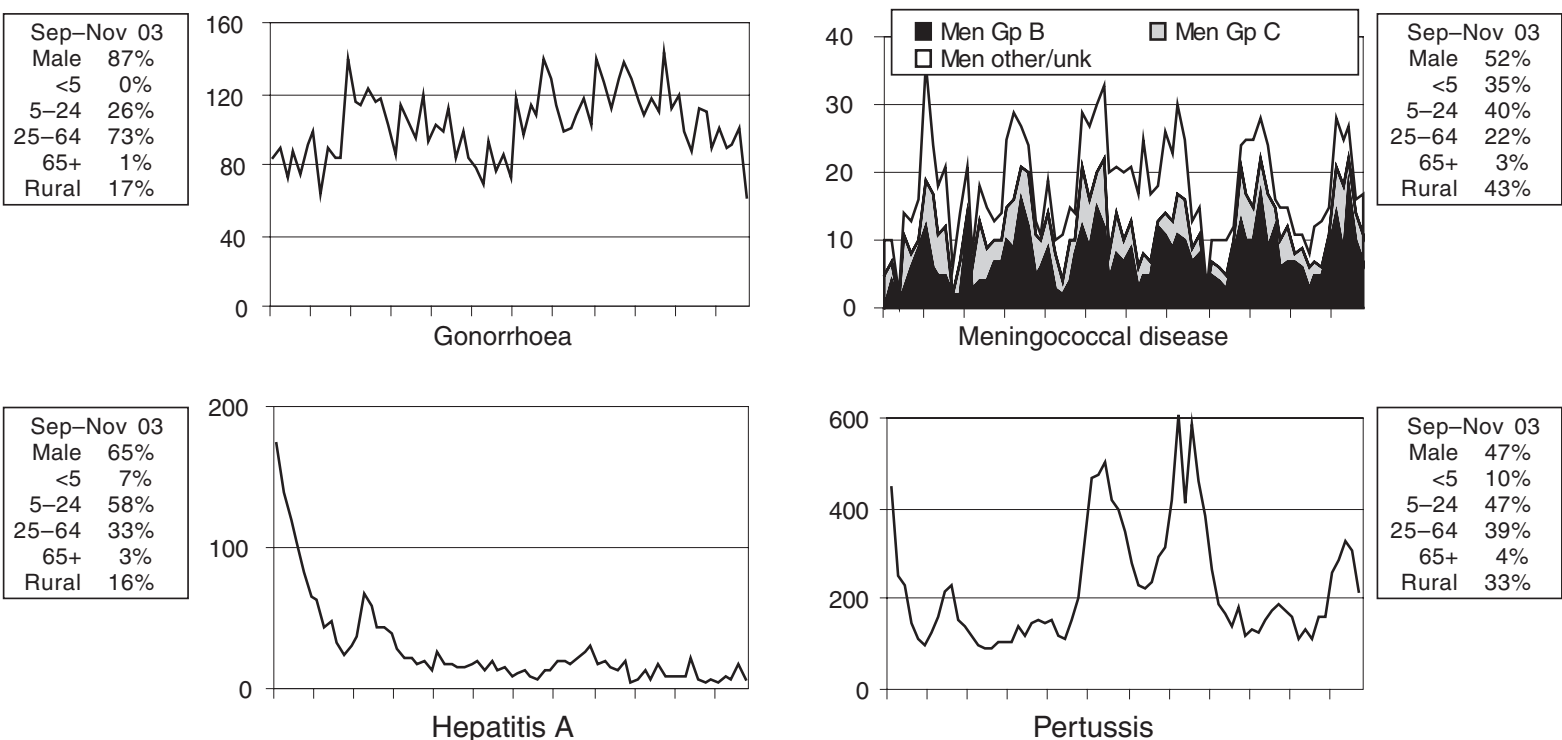

Pertussis

\begin{tabular}{|rc|}
\hline \multicolumn{2}{|c|}{ Sep-Nov 03 } \\
Male & $57 \%$ \\
$<5$ & $25 \%$ \\
$5-24$ & $6 \%$ \\
$25-64$ & $33 \%$ \\
$65+$ & $36 \%$ \\
Rural & $43 \%$ \\
\hline
\end{tabular}
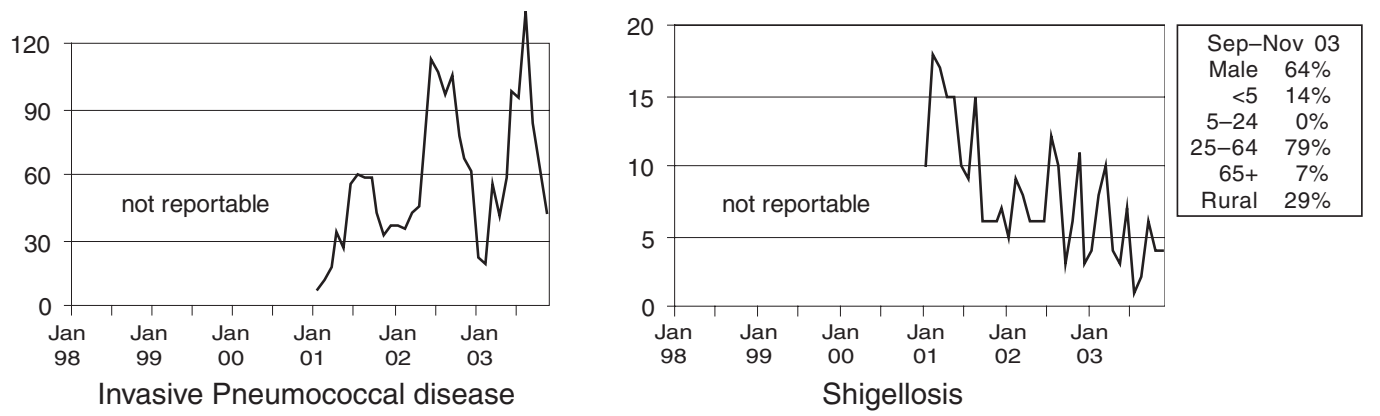


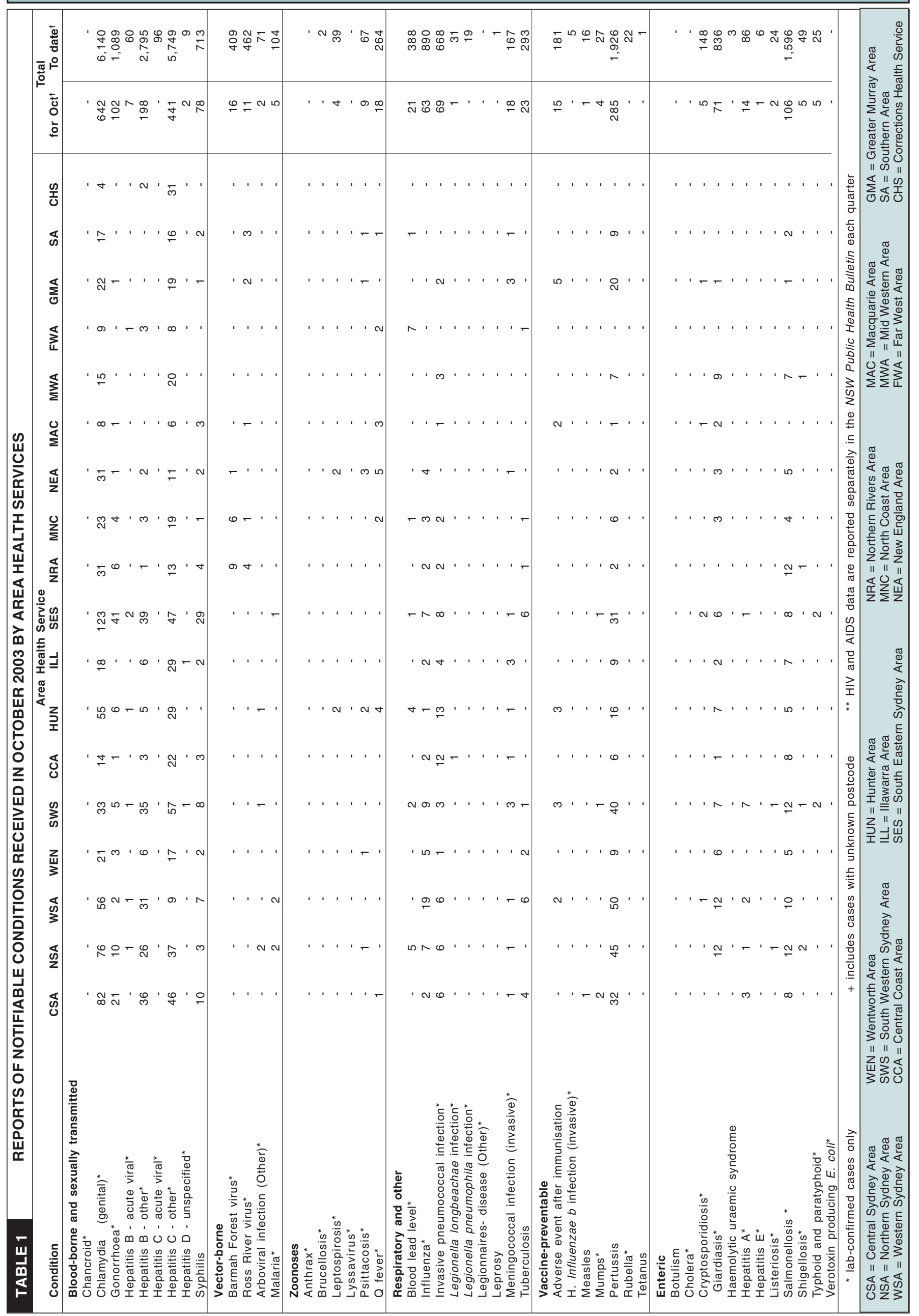




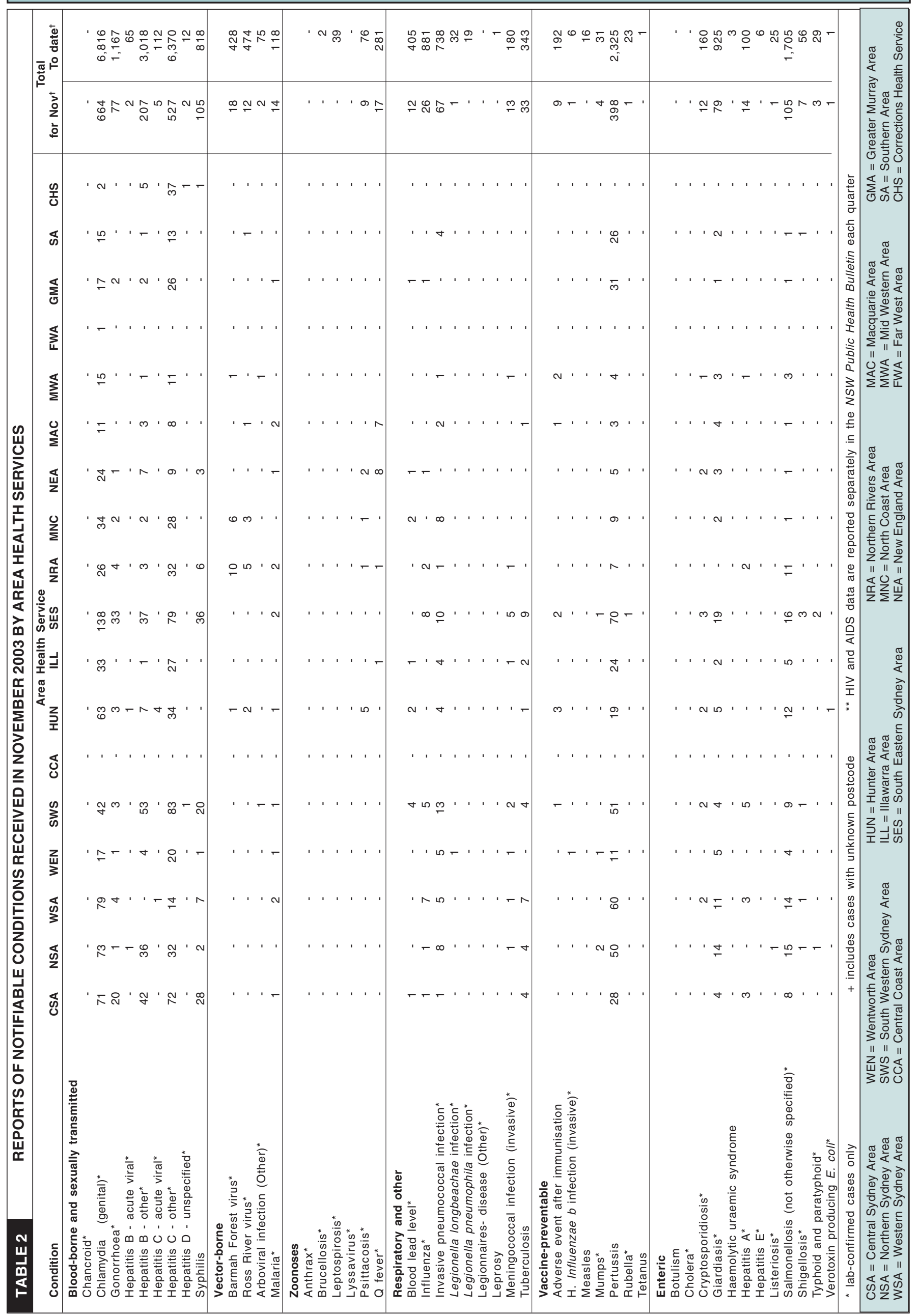

